Pacific

Journal of

Mathematics

\title{
ANISOTROPIC REAL CURVES AND BORDERED LINE ARRANGEMENTS
}

JohanNes Huisman and Michele Lattarulo 


\title{
ANISOTROPIC REAL CURVES AND BORDERED LINE ARRANGEMENTS
}

\author{
JOHANNES HUISMAN AND MiCHELE LATTARULO
}

\begin{abstract}
A real curve $X$ of genus $g \geq 2$ is anisotropic if the image of the canonical morphism $k: X \rightarrow \mathbb{P}^{g-1}$ is a rational real curve having no real points. We describe the moduli space of anisotropic curves, proving that it is isomorphic to the moduli space of double coverings of $\mathbb{P}^{2}$ ramified along real line arrangements.
\end{abstract}

\section{Introduction}

Let $X$ be a smooth projective real algebraic curve of genus $g$ greater than 1 . The canonical divisor on $X$ defines a morphism $k: X \rightarrow \mathbb{P}^{g-1}$ from $X$ into real projective space $\mathbb{P}^{g-1}$. The image $k(X)$ of $k$ is isomorphic either to $X$, or to the real projective line $\mathbb{P}^{1}$, or to the "empty circle" $\mathbb{S}^{1}$, understood as the smooth projective rational curve given by the affine equation $x^{2}+y^{2}=-1$. The real curve $X$ is said to be anisotropic if $k(X)$ is isomorphic to the empty circle.

Let $X$ be an anisotropic curve. The following facts are known [Gross and Harris 1981, Proposition 6.2]: the genus $g$ of $X$ is odd, its real locus $X(\mathbb{R})$ is empty, and $X$ may be represented, in affine 3 -space, by a pair of equations of the form $x^{2}+y^{2}=$ -1 and $w^{2}=p(x, y)$, where $p$ is a real polynomial of degree $g+1$. The canonical morphism $k$ can then be identified with the projection $X \ni(w, x, y) \mapsto(x, y)$.

The main problem in studying anisotropic curves is the absence of real points both in $X$ and in $k(X)$. To circumvent this, we proceed as follows. Let $X$ be defined by equations as above. We can consider the double covering of the plane, ramified along the $g+1$ real lines joining the $g+1$ pairs of complex conjugated branch points of $k$ in $\mathbb{S}^{1}$. This is a real surface $D$ having real points, and turns out to be tantamount to and easier to study than the original curve $X$.

Our results concern the moduli spaces of anisotropic curves. More precisely, we prove that there is an isomorphism between the moduli space of anisotropic curves and the moduli space of double coverings of $\mathbb{P}^{2}$ ramified along real line arrangements (see Theorem 7.1). As a corollary, we improve Proposition 6.2 of [Gross and Harris 1981], by showing, among other things, that any anisotropic

MSC2000: 14H15, 30F50, 52C30.

Keywords: anisotropic real curve, bordered line arrangement, moduli space. 
curve may be represented, in affine 3-space, by a pair of equations of the form $x^{2}+y^{2}=-1, w^{2}=p(x, y)$, where $p$ is a product of linear real polynomials (see Corollary 7.3). This is the analogue of the representation of complex hyperelliptic curves by equations of the form $y^{2}=q(x)$, where clearly, by the fundamental theorem of algebra, $q$ is a product of linear complex polynomials.

A different problem concerning anisotropic curves has been studied in [Cirre 2000], where the automorphism groups of anisotropic curves have been determined. Even though it is not our main subject here, it is interesting to examine the approach of that paper: there, anisotropic curves are studied as defined, in the affine plane, by complex equations of the form $y^{2}=q(x)$, where $q$ is a complex polynomial stable under the action of the antipodal antiholomorphic involution. A comparison between the two approaches is given in Example 8.1.

The paper is organized as follows. In Sections 2 and 3, we recall the facts we need about anisotropic curves and their moduli. In Sections 4-6, we study what we call bordered real line arrangements and the associated double covers of $\mathbb{P}^{2}$. Section 7 is the core of the paper. There we prove that the moduli space of real anisotropic curves is isomorphic to the moduli space of double covers of $\mathbb{P}^{2}$ ramified along real line arrangements. Finally, in Section 8, we apply our results to anisotropic curves of genus 3 .

For sake of simplicity, we defined projective curves by affine equations in this introduction. In the rest of the paper, we will use homogeneous equations, since this will make some statements nicer.

Convention. A variety over a field is supposed to be proper and geometrically integral [Hartshorne 1977], unless stated otherwise.

\section{Anisotropic curves}

Let $g$ be a natural integer greater than 1 . Let $X$ be a smooth real algebraic curve of genus $g$. The canonical divisor on $X$ defines a morphism $k: X \rightarrow \mathbb{P}^{g-1}$ from $X$ into the real projective space $\mathbb{P}^{g-1}$. Since $g \geq 2$, the image of $k$ is a real algebraic curve. Since the complexification of $k$ is the canonical map of the complexification of $X$, one sees that either $k$ is an isomorphism onto its image, or the image of $k$ is a smooth rational curve. In the latter case $X$ is said to be hyperelliptic.

Now, a smooth rational real algebraic curve is not necessarily isomorphic to the real projective line $\mathbb{P}^{1}$. Up to isomorphism, there are two real algebraic curves of genus 0: the real projective line $\mathbb{P}^{1}$ and the circle $\mathbb{S}^{1}$ in $\mathbb{P}^{2}$ given by the equation $x^{2}+y^{2}+z^{2}=0$.

Let $X$ be a real hyperelliptic curve, with canonical map $k$. The curve $X$ is said to be isotropic if $k(X)$ is isomorphic to the real projective line $\mathbb{P}^{1}$, and anisotropic if $k(X)$ is isomorphic to $\mathbb{S}^{1}$. 
The following statement is well known. We include a proof for convenience; see [Gross and Harris 1981, Proposition 6.1] for a different proof.

Proposition 2.1. The genus of an anisotropic curve is odd.

Proof. Let $X$ be a real hyperelliptic curve. Let $g$ be the genus of $X$, and let $k: X \rightarrow \mathbb{P}^{g-1}$ be the canonical map. Since $X$ hyperelliptic, the image curve $k(X)$ is of degree $g-1$. If $g$ is even, the degree of $k(X)$ is odd. It follows that the intersection of $k(X)$ with a real hyperplane of $\mathbb{P}^{g-1}$ contains a real point. In particular, $k(X)$ contains a real point, and hence is isomorphic to $\mathbb{P}^{1}$. Thus $X$ is isotropic if $g$ is even.

Let $X$ and $X^{\prime}$ be anisotropic curves. We say that $X$ and $X^{\prime}$ are isomorphic if there is an isomorphism $\alpha: X \rightarrow X^{\prime}$ of real algebraic curves. This precision may seem superfluous, but in the literature real curves are sometimes studied up to complex isomorphism. That is not the object of the present paper.

Let $g$ be an odd natural integer. Let $\mathcal{H}_{g}^{\text {an }}$ be the set of isomorphism classes of anisotropic curves of genus $g$. The set $\mathscr{H}_{g}^{a}$ is a subset of the moduli space $\mu_{g}^{\varnothing}$ of all smooth real curves of genus $g$ having no real points. Recall that $\mathcal{M}_{g}^{\varnothing}$ has a natural structure of a semianalytic variety [Huisman 1999]. It is natural to suspect that the subset $\mathscr{H}_{g}^{\text {an }}$ of $\mu_{g}^{\varnothing}$ is semianalytic, and indeed:

Proposition 2.2. The subset $\mathscr{H}_{g}^{\text {an }}$ of $\mathcal{M}_{g}^{\varnothing}$ is a semianalytic subset.

Proof. According to Proposition 2.1, we may assume that $g$ is odd. Let $X_{0}$ be a fixed anisotropic curve of genus $g$. Let $T\left(X_{0}\right)$ and $\operatorname{Mod}\left(X_{0}\right)$ be the real Teichmüller space and the modular group of $X_{0}$ (see [Huisman 2000] for details). By definition, the moduli space $\mu_{g}^{\varnothing}$ of all real curves of genus $g$ without real points is the semianalytic quotient $T\left(X_{0}\right) / \operatorname{Mod}\left(X_{0}\right)$.

Since $X_{0}$ is anisotropic, it admits a canonical involution [ -1$]$, as all hyperelliptic curves do. This involution induces a real analytic involution, again denoted by [-1], on $T\left(X_{0}\right)$. The set $S\left(X_{0}\right)$ of fixed points of [-1] on $T\left(X_{0}\right)$ is then a real analytic submanifold of $T\left(X_{0}\right)$, and its image in $\mu_{g}^{\varnothing}$ is the subset $\mathscr{H}_{g}^{\text {an }}$. It follows that $\mathcal{H}_{g}^{\text {an }}$ is a semianalytic subset of $\mu_{g}^{\text {an }}$.

To simplify the notation, we write $\mathscr{H}_{g}$ instead of $\mathscr{H}_{g}^{\text {an }}$ from now on.

\section{Branch loci of anisotropic curves}

Let $\mathbb{S}^{1}$ be the projective real algebraic curve in $\mathbb{P}^{2}$ defined by $x^{2}+y^{2}+z^{2}=0$. Since $\mathbb{S}^{1}$ is a rational normal curve in $\mathbb{P}^{2}$, each of its automorphisms extends uniquely to an automorphism of the real projective plane $\mathbb{P}^{2}$. Hence, one has an injective morphism

$$
\text { Aut } \mathbb{S}^{1} \rightarrow \text { Aut } \mathbb{P}^{2}=\mathrm{PGL}_{3}(\mathbb{R})
$$


We identify Aut $\mathbb{S}^{1}$ with its image. Let $\mathrm{PO}_{3}(\mathbb{R})$ be the image of the orthogonal group $O_{3}(\mathbb{R})$ in $\mathrm{PGL}_{3}(\mathbb{R})$, and $\mathrm{PSO}_{3}(\mathbb{R})$ the image of $\mathrm{SO}_{3}(\mathbb{R})$. We have

$$
\text { Aut } \mathbb{S}^{1}=\mathrm{PO}_{3}(\mathbb{R})=\mathrm{PSO}_{3}(\mathbb{R}) .
$$

Recall that a divisor $D$ on $\mathbb{S}$ is a finite formal sum $\sum m_{i} P_{i}$, where the $m_{i}$ are integers and the $P_{i}$ are closed points of $\mathbb{S}^{1}$. We may assume that $P_{i} \neq P_{j}$ if $i \neq j$. The degree of $D$ is equal to $2 \sum m_{i}$, since each closed point of $\mathbb{S}^{1}$ is of degree 2 . The divisor $D$ is effective if all $m_{i}$ are positive; it is reduced if all $m_{i}$ equal 1 .

Let $\tilde{\mathscr{P}}_{n}$ be the set of effective reduced divisors on $\mathbb{S}^{1}$ of degree $n$. Since $\mathbb{S}^{1}$ only carries divisors of even degree, the set $\tilde{\mathscr{B}}_{n}$ is nonempty only if $n$ is even. The set $\tilde{\mathscr{T}}_{2 n}$ has a natural structure of a connected real analytic manifold of dimension $2 n$. Indeed, the set of closed points of $\mathbb{S}^{1}$ can be identified with the set $\mathbb{S}^{1}(\mathbb{C}) / G$ of pairs of conjugate complex points of $\mathbb{S}^{1}$, where $G$ denotes the Galois group of $\mathbb{C} / \mathbb{R}$. Since $\mathbb{S}^{1}$ is a smooth conic, $\mathbb{S}^{1}(\mathbb{C})$ is the Riemann sphere. The action of $G$ on $\mathbb{S}^{1}(\mathbb{C})$ has no fixed points. Since this action is real analytic, $\mathbb{S}^{1}(\mathbb{C}) / G$ is a compact connected real analytic surface, in fact isomorphic to the real projective plane $\mathbb{P}^{2}(\mathbb{R})$. We already see that $\tilde{\mathscr{B}}_{2}$ has a natural structure of a connected real analytic manifold of dimension 2 . Now, let

$$
\Delta=\left\{\left(P_{1}, \ldots, P_{n}\right) \in \tilde{\mathscr{P}}_{2}^{n} \mid P_{i}=P_{j} \text { for some } i \neq j\right\} .
$$

be the big-diagonal in the $n$-fold power $\tilde{\mathscr{T}}_{2}^{n}$. Since $\tilde{\mathscr{P}}_{2}$ is a connected real analytic manifold of dimension 2 , the complement $\tilde{\mathscr{T}}_{2}^{n} \backslash \Delta$ of $\Delta$ is a connected real analytic manifold of dimension $2 n$. Let

$$
\mu: \tilde{\mathscr{P}}_{2}^{n} \backslash \Delta \rightarrow \tilde{\mathscr{P}}_{2 n}
$$

be the summation map defined by $\mu\left(P_{1}, \ldots, P_{n}\right)=\sum_{i=1}^{n} P_{i}$. The symmetric group $S_{n}$ acts on $\tilde{\mathscr{P}}_{2}^{n} \backslash \Delta$ without fixed points. Therefore, the quotient $\left(\tilde{\mathscr{B}}_{2}^{n} \backslash \Delta\right) / S_{n}$ is a connected real analytic manifold of dimension $2 n$. Since $\mu$ induces a bijection between that quotient manifold and the set $\tilde{\mathscr{R}}_{2 n}$, the latter set acquires a natural structure of a connected real analytic manifold of dimension $2 n$.

The automorphism group $\mathrm{PSO}_{3}(\mathbb{R})$ acts left-handedly on $\tilde{\mathscr{P}}_{2 n}$ by $\beta \cdot B=\beta(B)$,

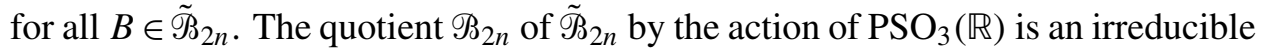
semianalytic variety of dimension $2 n-3$, if $n$ is even and greater than 2 [Huisman 1999]. One can check that in general $\tilde{\mathscr{T}}_{2 n}$ is a true semianalytic variety (that is, neither smooth nor analytic).

Let $g$ be an odd integer greater than 1. Let $X$ be an anisotropic curve of genus $g$, and let $k$ be the canonical map of $X$. After fixing an isomorphism between $k(X)$ and $\mathbb{S}^{1}$, we may and will assume that $k$ is a morphism from $X$ into $\mathbb{S}^{1}$. Let $B$ be the branch locus of $k$. Since $X$ is smooth, $B$ is a reduced effective divisor on $\mathbb{S}^{1}$. 
By Riemann-Hurwitz, the degree of $B$ is $2 g+2$. Define a map

$$
\lambda_{g}: \mathscr{H}_{g} \rightarrow \mathscr{B}_{2 g+2}
$$

by $\lambda(X)=B$; this is clearly well defined. One can check directly that $\lambda_{g}$ is analytic, but since the analyticity of $\lambda$ will also follow from our main result, we prefer to go on to the next section.

\section{Real line arrangements}

A real line arrangement in $\mathbb{P}^{2}$ is a reduced real algebraic curve in $\mathbb{P}^{2}$ all of whose irreducible components are real projective lines. We say that two real line arrangements $A$ and $A^{\prime}$ are isometric if there is $\beta \in \mathrm{PSO}_{3}(\mathbb{R})$ such that $\beta(A)=A^{\prime}$.

Let $n$ be a natural integer. Let $\tilde{A}_{n}$ be the set of all real line arrangements of degree $n$. The set $\tilde{A}_{n}$ has a natural structure of a connected real analytic manifold of dimension $2 n$. Indeed, let $\mathbb{R}[x, y, z]_{1}^{\star}$ be the set of all nonzero real linear forms in $x, y, z$. Let $\Gamma$ be the subset of the $n$-fold power $\mathbb{R}[x, y, z]_{1}^{\star n}$ of $\mathbb{R}[x, y, z]_{1}^{\star}$ given by

$$
\Gamma=\left\{\left(L_{1}, \ldots, L_{n}\right) \in \mathbb{R}[x, y, z]_{1}^{\star n} \mid L_{i}=\lambda L_{j} \text { for some } i \neq j \text { and some } \lambda \in \mathbb{R}^{\star}\right\} .
$$

Since $\mathbb{R}[x, y, z]_{1}^{\star}$ is a connected real analytic manifold of dimension 3 , the complement of $\Gamma$ in $\mathbb{R}[x, y, z]_{1}^{\star n}$ is a connected real analytic manifold of dimension $3 n$. Let $\mathbb{R}[x, y, z]_{n}^{\star}$ denote the set of nonzero homogeneous real polynomials of degree $n$ in $x, y, z$, and let

$$
\pi: \mathbb{R}[x, y, z]_{1}^{\star n} \backslash \Gamma \rightarrow \mathbb{R}[x, y, z]_{n}^{\star}
$$

be the product map defined by $\pi\left(L_{1}, \ldots, L_{n}\right)=\prod_{i=1}^{n} L_{i}$. The composition $Z \circ \pi$ maps $\left(L_{1}, \ldots, L_{n}\right)$ to the zero locus $Z\left(\prod L_{i}\right)$, which is a real line arrangement of degree $n$. It follows that the composition

$$
Z \circ \pi: \mathbb{R}[x, y, z]_{1}^{\star n} \backslash \Gamma \rightarrow \tilde{A}_{n}
$$

is a quotient map for the natural action of the semidirect product $\mathbb{R}^{\star n} \rtimes S_{n}$ on $\mathbb{R}[x, y, z]_{1}^{\star n} \backslash \Gamma$. Since that action is free, the quotient of $\mathbb{R}[x, y, z]_{1}^{\star n} \backslash \Gamma$ by $\mathbb{R}^{\star n} \rtimes S_{n}$ is a connected real analytic manifold of dimension $2 n$. Therefore, $\tilde{\mathscr{A}}_{n}$ acquires a natural structure of a connected real analytic manifold of dimension $2 n$. In fact, as for $\tilde{\mathscr{P}}_{2 n}$, the real analytic manifold $\tilde{\mathscr{A}}_{n}$ is naturally isomorphic to the quotient manifold $\left(\tilde{\mathscr{A}}_{1}^{n} \backslash \Delta\right) / S_{n}$, where $\Delta$ is the big diagonal in $\tilde{\mathscr{A}}_{1}^{n}$.

Let

$$
\tilde{\iota}_{n}: \tilde{\mathscr{A}}_{n} \rightarrow \tilde{\mathscr{B}}_{2 n}
$$

be the map that associates to a real line arrangement $A \in \tilde{A}_{n}$ the intersection product $A \cdot \mathbb{S}^{1}$. Note that, since $A$ is of degree $n$, the intersection product $A \cdot \mathbb{S}^{1}$ is a reduced effective divisor on $\mathbb{S}^{1}$ of degree $2 n$, i.e., one has, indeed, $\tilde{\iota}_{n}(A) \in \tilde{\mathscr{B}}_{2 n}$. 
Proposition 4.1. The map $\tilde{\iota}_{n}$ is an isomorphism of real analytic manifolds. Proof. Let

$$
\phi: \mathbb{R}[x, y, z]_{1}^{\star} \rightarrow \tilde{\mathscr{B}}_{2}
$$

be the map defined by setting $\phi(L)$ to the divisor $Z(L) \cdot \mathbb{S}^{1}$ on $\mathbb{S}^{1}$. It is easy to check that $\phi$ induces a real analytic isomorphism from $\mathbb{R}[x, y, z]_{1}^{\star} / \mathbb{R}^{\star}$ onto $\tilde{\mathscr{B}}_{2}$. If one identifies $\tilde{\mathscr{A}}_{1}$ with $\mathbb{R}[x, y, z]_{1}^{\star} / \mathbb{R}^{\star}$ as above, the induced isomorphism coincides with the map $\tilde{\iota}_{1}$. It follows that $\tilde{\iota}_{1}$ is a real analytic isomorphism. This proves the statement for $n=1$.

In order to prove the statement for arbitrary $n$, let

$$
\tilde{\iota}_{1}^{n}: \tilde{\mathscr{A}}_{1}^{n} \rightarrow \tilde{\mathscr{P}}_{2}^{n}
$$

be the $n$-fold cartesian power of $\tilde{\iota}_{1}$. Since $\tilde{\iota}_{1}$ is a real analytic isomorphism, the map $\tilde{\iota}_{1}^{n}$ is one too. Let, by abuse of notation, $\Delta$ denote the big diagonal in $\tilde{\mathscr{A}}_{1}^{n}$ as well as the big diagonal in $\tilde{\mathscr{S}}_{2}^{n}$. Then $\tilde{\iota}_{1}^{n}$ induces a real analytic isomorphism from $\left(\tilde{\mathscr{A}}_{1}^{n} \backslash \Delta\right) / S_{n}$ onto $\left(\tilde{\mathscr{B}}_{2}^{n} \backslash \Delta\right) / S_{n}$. As observed above, the former real analytic manifold can be identified with $\tilde{\mathscr{A}}_{n}$, and the latter with $\tilde{\mathscr{B}}_{2 n}$. The induced real analytic isomorphism from $\tilde{\mathscr{A}}_{n}$ into $\tilde{\mathscr{P}}_{2 n}$ clearly coincides with $\tilde{\iota}_{n}$. It follows that $\tilde{\iota}_{n}$ is a real analytic isomorphism.

Remark 4.2. The map $\tilde{\iota}_{n}$ can be naturally extended to a map from the set of possibly nonreduced real line arrangements of degree $n$ into the set of possibly nonreduced effective divisors of degree $2 n$ on $\mathbb{S}^{1}$. This extension is not a real analytic isomorphism, only a real analytic bijection [Huisman 2005, Theorem 2.1].

The group $\mathrm{PSO}_{3}(\mathbb{R})$ acts left-handedly on $\tilde{\mathscr{A}}_{n}$ by $\beta \cdot A=\beta(A)$. The quotient $\mathscr{A}_{n}=\mathrm{PSO}_{3}(\mathbb{R}) \backslash \tilde{A}_{n}$ is an irreducible semianalytic variety of dimension $2 n-3$, if $n$ is greater than 1 [Huisman 1999].

We recall that an analytic map between semianalytic varieties is a morphism between locally ringed spaces. It is then also clear what is understood by an isomorphism of semianalytic varieties.

Corollary 4.3. The map $\tilde{\iota}_{n}$ is equivariant and induces an isomorphism

$$
\iota_{n}: \mathscr{A}_{n} \rightarrow \mathscr{B}_{2 n}
$$

of semianalytic varieties.

\section{Bordered real line arrangements}

A bordered real line arrangement is a pair $(A, O)$, where $A$ is a real line arrangement and $O$ is a closed subset of $\mathbb{P}^{2}(\mathbb{R})$ whose boundary is equal to $A(\mathbb{R})$. Let $(A, O)$ and $\left(A^{\prime}, O^{\prime}\right)$ be two bordered real line arrangements. Then $(A, O)$ and $\left(A^{\prime}, O^{\prime}\right)$ are isometric if there is $\beta \in \mathrm{PSO}_{3}(\mathbb{R})$ such that $\beta(A)=A^{\prime}$ and $\beta(O)=O^{\prime}$. 


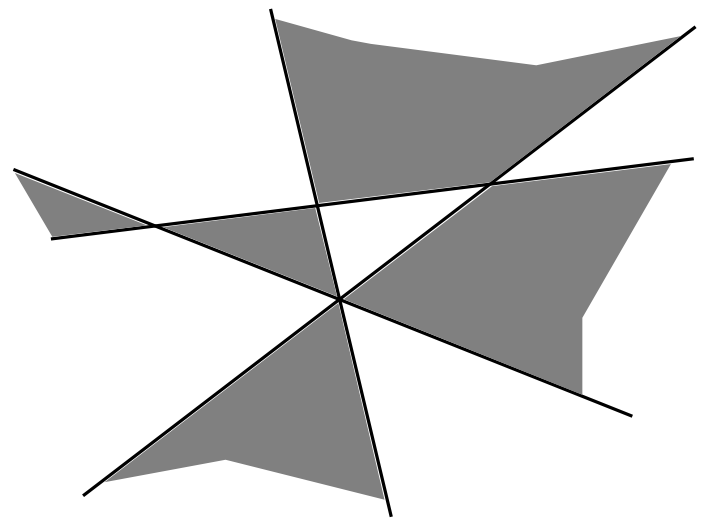

Figure 1. A bordered real line arrangement of degree 4 .

Proposition 5.1. Let $A$ be a real line arrangement. There is a closed subset $O$ of $\mathbb{P}^{2}(\mathbb{R})$ such that $(A, O)$ is a bordered real line arrangement if and only if $\operatorname{deg} A$ is even.

Proof. Suppose that $\operatorname{deg} A$ is even. Let $F$ be a homogeneous real polynomial defining $A$. The subset $O$ defined as the locus of all points of $\mathbb{P}^{2}(\mathbb{R})$ where $F$ is nonnegative, is well defined. It is clear that $(A, O)$ is a bordered real line arrangement.

To prove the converse, let $O$ be a closed subset of $\mathbb{P}^{2}(\mathbb{R})$ such that $(A, O)$ is a bordered real line arrangement. Let $F$ be a homogeneous real polynomial defining $A$. We have to show that $\operatorname{deg} F$ is even. Let $\pi: S^{2} \rightarrow \mathbb{P}^{2}(\mathbb{R})$ be the canonical map from the 2-sphere $S^{2}$ into $\mathbb{P}^{2}(\mathbb{R})$. The closed subsets of $S^{2}$ given by

$$
C_{+}=\{F \circ \pi \geq 0\} \quad \text { and } \quad C_{-}=\{F \circ \pi \leq 0\}
$$

are well defined. Since the boundaries of $\pi^{-1}(O), C_{+}$and $C_{-}$are all equal to the zero set of $F \circ \pi$, the subset $\pi^{-1}(O)$ is equal to $C_{+}$or $C_{-}$. It follows that either $C_{+}$or $C_{-}$is stable under multiplication by -1 on $S^{2}$. Therefore, the degree of $F$ is even.

Let $n$ be a natural integer. Let $\tilde{\mathscr{D}}_{n}$ be the set of all bordered real line arrangements of degree $n$. By the preceding proposition, the set $\tilde{\mathscr{D}}_{n}$ is nonempty if and only if $n$ is even. The set $\tilde{\mathscr{D}}_{n}$ has a natural structure of an irreducible real analytic manifold of dimension $2 n$, if $n$ is even. Indeed, let

$$
\gamma_{n}: \mathbb{R}[x, y, z]_{1}^{\star n} \backslash \Gamma \rightarrow \tilde{\mathscr{D}}_{n}
$$

be the map defined by $\gamma_{n}\left(L_{1}, \ldots, L_{n}\right)=(A, O)$, where $A$ is the real line arrangement defined by $\prod L_{i}$ and $O$ is the closed subset $\left\{\prod L_{i} \geq 0\right\}$ of $\mathbb{P}^{2}(\mathbb{R})$. Let $H$ be the subgroup of $\mathbb{R}^{\star n}$ of all elements $\left(\lambda_{1}, \ldots, \lambda_{n}\right)$ for which $\prod \lambda_{i}$ is positive. The 
natural action of $\mathbb{R}^{\star n} \rtimes S_{n}$ on $\mathbb{R}[x, y, z]_{1}^{\star n} \backslash \Gamma$ restricts to an action of $H \rtimes S_{n}$. The map $\gamma_{n}$ induces a bijection between the quotient $\left(\mathbb{R}[x, y, z]_{1}^{\star n} \backslash \Gamma\right) / H \rtimes S_{n}$ and $\tilde{\mathscr{D}}_{n}$. Since the former subset is a connected real analytic manifold of dimension $2 n$, the set $\tilde{\mathscr{D}}_{n}$ acquires the structure of such a manifold by transport of structure.

One has a forgetful map

$$
\tilde{\phi}_{n}: \tilde{\mathscr{D}}_{n} \rightarrow \tilde{\mathscr{A}}_{n}
$$

that associates to a bordered real line arrangement $(A, O)$ the real line arrangement $A$. The map $\tilde{\phi}_{n}$ is clearly an unramified real analytic covering of $\tilde{\mathscr{A}}_{n}$ of degree 2. The group $\mathrm{PSO}_{3}(\mathbb{R})$ acts left-handedly on $\tilde{\mathscr{D}}_{n}$ by $\beta \cdot(A, O)=(\beta(A), \beta(O))$. Let

$$
\mathscr{D}_{n}=\mathrm{PSO}_{3}(\mathbb{R}) \backslash \tilde{D}_{n} .
$$

Then $\mathscr{D}_{n}$ is an irreducible semianalytic variety of dimension $2 n-3$, if $n$ is even and greater than 1 . Since $\tilde{\phi}_{n}$ is equivariant, one has an induced real analytic map

$$
\phi_{n}: \mathscr{D}_{n} \rightarrow \mathscr{A}_{n}
$$

Note that $\phi_{n}$ is not necessarily unramified.

\section{Double coverings ramified along a line arrangement}

Let $f: D \rightarrow \mathbb{P}^{2}$ be a ramified double covering of $\mathbb{P}^{2}$, where $D$ is a not necessarily smooth real algebraic variety. We say that $f$ is ramified along a real line arrangement if the ramification locus of $f$ is a real line arrangement in $\mathbb{P}^{2}$. Two such coverings $f: D \rightarrow \mathbb{P}^{2}$ and $f^{\prime}: D^{\prime} \rightarrow \mathbb{P}^{2}$ are said to be isometric if there is an isomorphism $\alpha: D \rightarrow D^{\prime}$ and an isometry $\beta$ of $\mathbb{P}^{2}$ such that $f^{\prime} \circ \alpha=\beta \circ f$.

Let $f: D \rightarrow \mathbb{P}^{2}$ be a double covering ramified along a real line arrangement. Let $A$ be the real line arrangement along which $f$ is ramified. Let $O=f(D(\mathbb{R}))$. Then $(A, O)$ is a bordered real line arrangement. Moreover, any bordered real line arrangement is the bordered real line arrangement of a double covering of $\mathbb{P}^{2}$ ramified along a real line arrangement. It follows that there is a natural bijection between the set of isometry classes of bordered real line arrangements of degree $n$, and the set of isometry classes of double coverings of $\mathbb{P}^{2}$ that are ramified along a real line arrangement of degree $n$. In the sequel, we will identify the latter set with the former, and speak about elements of $\mathscr{D}_{n}$ as ramified double coverings of $\mathbb{P}^{2}$. In particular, the set of isometry classes of double coverings of $\mathbb{P}^{2}$ that are ramified along a real line arrangement of degree $n$ carries a natural structure of an irreducible semianalytic variety of dimension $2 n-3$, if $n$ is even and greater than 1 . The forgetful map

$$
\phi_{n}: \mathscr{D}_{n} \rightarrow \mathscr{A}_{n}
$$

associates to the isometry class of a double covering in $\mathscr{D}_{n}$, its ramification locus. 


\section{Moduli of anisotropic curves}

Let $g$ be an odd natural integer greater than 1 . Recall that $\mathscr{H}_{g}$ is the semianalytic moduli space of anisotropic curves of genus $g$. Define a map

$$
\rho_{g}: \mathscr{D}_{g+1} \rightarrow \mathscr{H}_{g}
$$

as follows. Let $f: D \rightarrow \mathbb{P}^{2}$ be a double covering, ramified along a real line arrangement of degree $g+1$. Let $X$ be the fiber product of $D$ and $\mathbb{S}^{1}$ over $\mathbb{P}^{2}$ with respect to the morphism $f: D \rightarrow \mathbb{P}^{2}$ and the inclusion morphism $\mathbb{S}^{1} \hookrightarrow \mathbb{P}^{2}$, i.e.,

$$
X=D \times_{\mathbb{P}^{2}} \mathbb{S}^{1} .
$$

The curve $X$ is a smooth real algebraic curve. The morphism $f$ induces a morphism

$$
h: X \rightarrow \mathbb{S}^{1} \text {. }
$$

Since $f$ is a double covering, the degree of $h$ is equal to 2. Since the ramification locus of $h$ is a reduced divisor of degree $2(g+1)$ on $\mathbb{S}^{1}$, the curve $X$ is a smooth real algebraic curve of genus $g$. Since $X$ admits a morphism of degree 2 into $\mathbb{S}^{1}$, the curve $X$ is an anisotropic curve. Define $\rho_{g}(D)=X$. It is clear that $\rho_{g}$ is well defined.

We have a commutative diagram

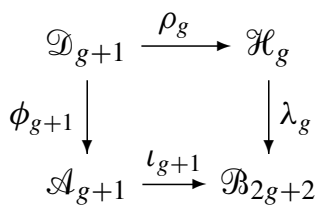

Theorem 7.1. Let $g$ be an odd natural integer greater than 1 . The map $\rho_{g}$ is a real analytic isomorphism of semianalytic spaces.

Proof. We first prove surjectivity. Let $X$ be an anisotropic curve of genus $g$, and let $k$ be the canonical map of $X$. Since $X$ is anisotropic, we may assume that $k$ is a morphism of degree 2 from $X$ into $\mathbb{S}^{1}$. Let $B$ be the branch locus of $k$. Since $\tilde{\iota}_{g+1}$ is surjective, there is a real line arrangement $A$ of degree $g+1$ such that $A \cdot \mathbb{S}^{1}=B$. Since $g$ is odd (Proposition 2.1), $g+1$ is even and there is a double covering $f: D \rightarrow \mathbb{P}^{2}$ ramified along $A$. Let $f^{-}: D^{-} \rightarrow \mathbb{P}^{2}$ be the double covering of $\mathbb{P}^{2}$ obtained from $f$ by twisting the real structure [Huisman and Lattarulo 2005]. Then either the curve $D \times_{\mathbb{P}^{2}} \mathbb{S}^{1}$ or the curve $D^{-} \times_{\mathbb{P}^{2}} \mathbb{S}^{1}$ is isomorphic to $X$, i.e.,

$$
\rho_{g}(D)=X \quad \text { or } \quad \rho_{g}\left(D^{-}\right)=X .
$$

This proves that $\rho_{g}$ is surjective.

Next, we prove the injectivity of $\rho_{g}$. Let $f: D \rightarrow \mathbb{P}^{2}$ and $f^{\prime}: D^{\prime} \rightarrow \mathbb{P}^{2}$ be double coverings that are ramified along the real line arrangements $A$ and $A^{\prime}$, 
respectively, both of degree $g+1$. Let $(A, O)$ and $\left(A^{\prime}, O^{\prime}\right)$ be the bordered real line arrangements associated to $f$ and $f^{\prime}$. Let $F$ and $F^{\prime}$ be homogeneous polynomials in $\mathbb{R}[x, y, z]$ of degree $g+1$ that define the bordered real line arrangements $(A, O)$ and $\left(A^{\prime}, O^{\prime}\right)$. This means that $A$ is the vanishing set of $F$ in $\mathbb{P}^{2}$ and $O$ is the set of real points of $\mathbb{P}^{2}$ at which $F$ is nonnegative, and similarly for $\left(A^{\prime}, O^{\prime}\right)$ and $F^{\prime}$. Then $D$ is isomorphic to the ramified double covering of $\mathbb{P}^{2}$ given by the equation $w^{2}=F(x, y, z)$, and similarly for $D^{\prime}$.

Let $X=D \times_{\mathbb{P}^{2}} \mathbb{S}^{1}$ and $X^{\prime}=D^{\prime} \times_{\mathbb{P}^{2}} \mathbb{S}^{1}$ be the anisotropic curves induced by $D$ and $D^{\prime}$. The curve $X$ is isomorphic to the ramified double covering of $\mathbb{S}^{1}$ defined by the equation $w^{2}=F(x, y, z)$, and similarly for $X^{\prime}$. Here there is a slight abuse of notation: we denote again by $F$ its restriction to $\mathbb{S}^{1}$.

Assume that $X$ and $X^{\prime}$ are isomorphic via $\alpha: X \rightarrow X^{\prime}$. There is an automorphism $\beta: \mathbb{S}^{1} \rightarrow \mathbb{S}^{1}$ such that $h^{\prime} \circ \alpha=\beta \circ h$, where $h$ and $h^{\prime}$ are the morphisms induced by $f$ and $f^{\prime}$. Since $\mathbb{S}^{1}$ is a rational normal curve in $\mathbb{P}^{2}, \beta$ is the restriction of an isometry of $\mathbb{P}^{2}$, again denoted by $\beta$. Since $\beta$ maps the branch locus of $f$ into the branch locus of $f^{\prime}$, there is a nonzero real number $\mu$ such that $\beta^{\star}\left(F^{\prime}\right)=\mu F$. In particular, $\beta(A)=A^{\prime}$. Over $\mathbb{S}^{1}$, one has

$$
\left(\alpha^{\star}\left(w^{\prime}\right)\right)^{2}=\alpha^{\star}\left(\left(w^{\prime}\right)^{2}\right)=\beta^{\star}\left(F^{\prime}\right)=\mu F .
$$

Hence, the rational function $\alpha^{\star}\left(w^{\prime}\right) / w$ on $X$ is a square root of $\mu$. Since $X$ is geometrically irreducible, $\mu$ is positive. It follows that $\beta \cdot(O)=O^{\prime}$, i.e., $D$ and $D^{\prime}$ are isometric. This proves that $\rho_{g}$ is injective, and therefore, bijective.

It remains to show that $\rho_{g}$ and $\rho_{g}^{-1}$ are analytic. To do this for $\rho_{g}$, let $U$ be the universal family of double covers over $\tilde{\mathscr{D}}_{g+1}$. More precisely, $U$ is a relative real algebraic surface over the real analytic manifold $\tilde{\mathscr{D}}_{g+1}$, which is endowed with a morphism $f$ over $\tilde{\mathscr{D}}_{g+1}$ from $U$ into $\mathbb{P}^{2} \times \tilde{\mathscr{D}}_{g+1}$ such that, for all $D \in \tilde{\mathscr{D}}_{g+1}$, the fiber $U_{D}$ of $U$ is equal to $D$, and the fiber $f_{D}$ of $f$ is the ramified cover $D \rightarrow \mathbb{P}^{2}$.

Let $V$ be the fiber product of $U$ and $\mathbb{S}^{1} \times \tilde{\mathscr{D}}_{g+1}$ over $\mathbb{P}^{2} \times \tilde{\mathscr{D}}_{g+1}$. Then $V$ is a real analytic family of anisotropic curves of genus $g$ over $\tilde{\mathscr{D}}_{g+1}$. By the universal property of the moduli space $\mathcal{H}_{g}$, there is a unique real analytic map $\tilde{\rho}_{g}$ from $\tilde{\mathscr{D}}_{g+1}$ into $\mathcal{H}_{g}$ such that $\tilde{\rho}_{g}(D)$ is equal to the fiber $V_{D}$ of $V$ at $D$. It follows that $\tilde{\rho}_{g}$ induces a real analytic map from $\mathscr{D}_{g+1}$ into $\mathscr{H}_{g}$. It is clear that the induced map coincides with $\rho_{g}$. Therefore, $\rho_{g}$ is real analytic.

A similar argument will prove that $\rho_{g}^{-1}$ is real analytic, once one has a good universal family of anisotropic curves of genus $g$. Therefore, we will only indicate the construction of that universal family.

Recall the notation introduced in the proof of Proposition 2.2: $X_{0}$ is a fixed anisotropic curve of genus $g, T\left(X_{0}\right)$ the real Teichmüller space of $X_{0}, \operatorname{Mod}\left(X_{0}\right)$ the modular group of $X_{0}$, and $S\left(X_{0}\right)$ the subset of $T\left(X_{0}\right)$ of fixed points for the induced action of the hyperelliptic involution [-1] of $X_{0}$ on $T\left(X_{0}\right)$. 
Unlike $\mu_{g}^{\varnothing}$, the Teichmüller space $T\left(X_{0}\right)$ admits a real analytic universal family $\mathscr{L}$ of real curves of genus $g$ without real points. Let $\mathscr{Y}$ be the restriction to $S\left(X_{0}\right)$ of the universal family $\mathscr{X}$. The involution $[-1]$ on the fiber $X_{0}$ of $\mathscr{Y}$ extends to an involution of $y$ over $S\left(X_{0}\right)$ that we again denote by [-1]. The space $S\left(X_{0}\right)$ is a Teichmüller space of anisotropic curves of genus $g$. The quotient family $\mathscr{Y} /[-1]$ is a real analytic family of smooth rational curves without real points over $S\left(X_{0}\right)$. Since $S\left(X_{0}\right)$ is contractible, the family $9 /[-1]$ is isomorphic to the constant family $\mathbb{S}^{1} \times S\left(X_{0}\right)$. Hence, we have a universal family $9 y$ of anisotropic curves over a real analytic manifold $S\left(X_{0}\right)$, endowed with a morphism $f$ over $S\left(X_{0}\right)$ from 9 into $\mathbb{S}^{1} \times S\left(X_{0}\right)$. As above, this family induces a real analytic map from $S\left(X_{0}\right)$ into $\mathscr{D}_{g+1}$ that, in turn, induces a real analytic map that coincides with $\rho_{g}^{-1}$.

Corollary 7.2. Let $X$ and $X^{\prime}$ be anisotropic curves. Let $(A, O)$ and $\left(A^{\prime}, O^{\prime}\right)$ be the bordered real line arrangements corresponding to $X$ and $X^{\prime}$ respectively. Then $X$ and $X^{\prime}$ are isomorphic if and only if $(A, O)$ and $\left(A^{\prime}, O^{\prime}\right)$ are isometric.

As a consequence of Theorem 7.1 we get explicit formulas for anisotropic curves, and are able to determine when two such curves are isomorphic:

Corollary 7.3. Let $f: X \rightarrow \mathbb{S}^{1}$ be an anisotropic curve of genus $g$. There are linear forms $L_{1}, \ldots, L_{g+1} \in \mathbb{R}[x, y, z]$, with $L_{i}$ not a multiple of $L_{j}$ for $i \neq j$, such that the curve $X$ is the normalization of the real curve in $\mathbb{P}^{3}$ defined by the equations

$$
x^{2}+y^{2}+z^{2}=0 \quad \text { and } \quad w^{2} z^{g-1}=p(x, y, z),
$$

where $p=L_{1} \cdots L_{g+1}$. If $L_{1}^{\prime}, \ldots, L_{g+1}^{\prime}$ are other linear forms, with $L_{i}^{\prime}$ not a multiple of $L_{j}^{\prime}$ for $i \neq j$, then the anisotropic curve $X^{\prime}$ defined by $L_{1}^{\prime}, \ldots, L_{g+1}^{\prime}$ as above is isomorphic to $X$ if and only if there are nonzero real numbers $\mu_{1}, \ldots, \mu_{g+1}$, an isometry $\beta \in \mathrm{PSO}_{3}(\mathbb{R})$, and a permutation $\sigma \in S_{g+1}$ such that

$$
\beta^{\star}\left(L_{i}\right)=\mu_{i} L_{\sigma(i)} \quad \text { and } \quad \mu_{1} \cdots \mu_{g+1}>0 .
$$

\section{Examples: anisotropic curves of genus 3}

We now apply our results to the study of anisotropic curves of genus 3. In Example 8.1 we determine the anisotropic curves with automorphism group $S_{4} \times \mathbb{Z} / 2 \mathbb{Z}$. In Example 8.2 we show that an anisotropic curve defined by a bordered line arrangement $(A, O)$ is not necessarily isomorphic to the opposite anisotropic curve $X^{-}$ defined by the opposite bordered line arrangement $\left(A, \mathbb{P}^{2}(\mathbb{R}) \backslash O^{\circ}\right)$. Finally, in Example 8.3 we show how our methods apply to the study of moduli spaces of anisotropic curves with a fixed automorphism group.

Example 8.1. Suppose that $X$ is an anisotropic curve with automorphism group isomorphic to $S_{4} \times \mathbb{Z} / 2 \mathbb{Z}$. Let $(A, O)$ be the bordered line arrangement associated 


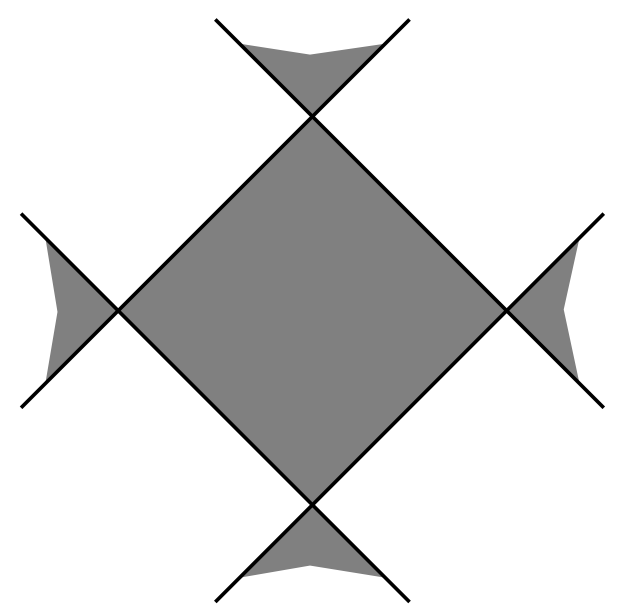

Figure 2. One of the two bordered real line arrangements $(A, O)$ of Example 8.1 with isometry group $S_{4}$.

to $X$. Then the group of isometries of $(A, O)$ is isomorphic to $S_{4}$. One may assume that the group of isometries of $(A, O)$ is generated by the automorphism $\beta_{1}$ of order 4 and the involution $\beta_{2}$ defined by

$$
\beta_{1}([x: y: z])=[-y: x: z] \quad \beta_{2}([x: y: z])=[z: y: x]
$$

and that $A$ contains the real projective line defined by $x+y+z=0$. Put

$$
p(x, y, z)=(x+y+z)(x-y+z)(-x+y+z)(-x-y+z) .
$$

Then $A$ is defined by the polynomial $p$, and $O$ is either equal to $\{p \geq 0\}$, or $\{p \leq 0\}$. It follows that $X$ is isomorphic to the anisotropic curve of $\mathbb{P}^{3}$ defined, over $\mathbb{S}^{1}$, either by the equation $w^{2} z^{g-1}=p(x, y, z)$ or by the equation $w^{2} z^{g-1}=$ $-p(x, y, z)$. Moreover the automorphisms group of $X$ is isomorphic to $S_{4} \times \mathbb{Z} / 2 \mathbb{Z}$ : this follows by the considerations above, and by the fact that the order of Aut $X$ cannot exceed 48, since the genus of $X$ is 3 .

We take the occasion to compare with the approach followed in [Cirre 2000]. There, anisotropic curves are studied as curves defined, in the affine plane, by complex equations of the form $v^{2}=q(u)$, stable under the action of the antipodal antiholomorphic involution $\sigma(u, v)=\left(-1 / \bar{u}, \bar{v} \sqrt{q(0)} / \bar{u}^{g+1}\right)$. Doing it like this, one obtains the following equation for one of the anisotropic curves with isomorphism group $S_{4} \times \mathbb{Z} / 2 \mathbb{Z}$ : $v^{2}=\left(u^{2}+(-1+i) u+i\right)\left(u^{2}+(1+i) u-i\right)\left(u^{2}+(1-i) u+i\right)\left(u^{2}+(-1-i) u-i\right)$ 
(the four factors correspond to the four factors of the polynomial $p$ above) and the automorphism group of $X$ is generated by the hyperelliptic involution and by the automorphisms

$$
\gamma_{1}(u, v)=(i u, v) \quad \gamma_{2}(u, v)=\left(\frac{-u+1}{u+1}, \frac{-4 v}{(u+1)^{4}}\right)
$$

that correspond to the automorphisms $\beta_{1}$ and $\beta_{2}$ above.

Example 8.2. Let $X$ be the anisotropic curve defined in $\mathbb{P}^{3}$ by an equation of the form $w^{2} z^{g-1}=p(x, y, z)$ over $\mathbb{S}^{1}$. The curve $X^{-}$defined by the equation $w^{2} z^{g-1}=-p(x, y, z)$ over $\mathbb{S}^{1}$ is said to be its opposite curve. It is clear that $X$ and $X^{-}$have the same branch locus and isomorphic automorphism groups, so it is natural to ask when $X$ and $X^{-}$are isomorphic. This question is of central interest when one studies the problem of characterizing the Jacobians of real curves among all principally polarized real abelian varieties; see [Huisman and Lattarulo 2005; Lattarulo 2003]. We will give a complete answer in a forthcoming paper. Here we show that $X$ and $X^{-}$may be nonisomorphic. In Example 8.3 we will see that $X$ and $X^{-}$may be isomorphic.

Let $X$ be one of the curves of Example 8.1. If there were an isomorphism $\gamma: X \rightarrow X^{-}$, its complexification $\alpha=\gamma_{\mathbb{C}}$ would be a nonreal automorphism of $X_{\mathbb{C}}$. But we have seen in Example 8.1 that Aut $X=S_{4} \times \mathbb{Z} / 2 \mathbb{Z}$. Since Aut $X$ is a subgroup of Aut $X_{\mathbb{C}}$ and since a complex curve of genus 3 cannot have more than 48 automorphisms, we conclude that Aut $X_{\mathbb{C}}=$ Aut $X$. Thus all the automorphisms of $X_{\mathbb{C}}$ are real for $X$ and, by the remarks above, $X$ and $X^{-}$cannot be isomorphic.

Example 8.3. Let $G$ be a finite group and let $\mathscr{H}_{g}(G)$ be the subspace of $\mathscr{H}_{g}$ consisting of the anisotropic curves $X \in \mathscr{H}_{g}$ such that $G \times \mathbb{Z} / 2 \mathbb{Z}$ is isomorphic to a subgroup of Aut $X$. The theory developed in this paper allows to answer to the following questions:

(1) When is $\mathscr{H}_{g}(G)$ nonempty? (This question has also been treated in [Cirre 2000].)

(2) What is the real dimension of $\mathscr{H}_{g}(G)$ as a subspace of $\mathscr{H}_{g}$ ?

(3) What is the number of connected components of $\mathscr{H}_{g}(G)$ ?

For example, in genus 3 , one easily obtains the following table, which summarizes the answers to our questions. The first row lists the groups $G$ for which $\mathscr{H}_{3}(G)$ is nonempty. The second and the third rows show the dimension and the number of connected components of $\mathscr{H}_{3}(G)$, respectively.

\begin{tabular}{|c|cccccc|}
\hline$G$ & $S_{4}$ & $D_{4}$ & $D_{3}$ & $D_{2}$ & $\mathbb{Z} / 2 \mathbb{Z}$ & Id \\
\hline $\operatorname{dim} \mathscr{H}_{3}(G)$ & 0 & 1 & 1 & 2 & 3 & 5 \\
$\# \pi_{0} \mathscr{H}_{3}(G)$ & 2 & 3 & 1 & 3 & 5 & 1 \\
\hline
\end{tabular}


Here we describe in detail the moduli space $\mathscr{H}_{3}\left(D_{4}\right)$. The moduli space $\mathscr{H}_{3}\left(S_{4}\right)$ has essentially been described in Example 8.1. For the remaining cases (and in higher genus) one proceeds in a similar way.

So, let $X \in \mathscr{H}_{3}\left(D_{4}\right)$. We choose as generators of $D_{4}$, embedded in $\mathrm{PO}_{3}(\mathbb{R})$, the isometries of $\mathbb{P}^{2}$ given by

$$
\beta_{1}[x: y: z]=[x:-y: z], \quad \beta_{2}[x: y: z]=[-y: x: z] .
$$

The bordered line arrangements whose group of isometries contains $D_{4}$ are $(\{p=$ $0\},\{p \geq 0\})$, where $p \in C_{1} \cup C_{2} \cup C_{3}$ and

$$
\begin{aligned}
& C_{1}=\left\{p \in \mathbb{R}[x, y, z]: p(x, y, z)=\left(x^{2}-a y^{2}\right)\left(a x^{2}-y^{2}\right), \quad a \in \mathbb{R}^{\star,+}, a<1\right\}, \\
& C_{2}=\left\{p \in \mathbb{R}[x, y, z]: p(x, y, z)=\left(x^{2}-b z^{2}\right)\left(y^{2}-b z^{2}\right), \quad b \in \mathbb{R}^{\star,+}\right\}, \\
& C_{3}=\left\{p \in \mathbb{R}[x, y, z]: p(x, y, z)=-\left(x^{2}-b z^{2}\right)\left(y^{2}-b z^{2}\right), b \in \mathbb{R}^{\star,+}\right\} .
\end{aligned}
$$

It follows that $X$ may be defined, in $\mathbb{P}^{3}$, by the equation $w^{2} z^{g-1}=p(x, y, z)$ over $\mathbb{S}^{1}$, where $p \in C_{1} \cup C_{2} \cup C_{3}$. More precisely, let $\rho$ be the map that sends a polynomial $p \in C_{1} \cup C_{2} \cup C_{3}$ into the anisotropic curve $X \in \mathscr{H}_{3}\left(D_{4}\right)$ defined, in $\mathbb{P}^{3}$, by the equation $w^{2} z^{g-1}=p(x, y, z)$ over $\mathbb{S}^{1}$. Then $\rho$ is a bijection, the 3 connected components of $\mathscr{H}_{3}\left(D_{4}\right)$ are $\rho\left(C_{1}\right), \rho\left(C_{2}\right), \rho\left(C_{3}\right)$, and each one of these components is one-dimensional.

We remark that, if $p \in C_{1}$ is the polynomial corresponding to the value $a=$ $3-2 \sqrt{2}$, the curve $X=\rho(p)$ is isomorphic to its opposite curve $X^{-}$(see Example 8.2 for this notion). If $q \in C_{2}$ is the polynomial corresponding to the value $b=2$, the curve $X=\rho(q)$ and its opposite $X^{-}$are the curves studied in Examples 8.1 and 8.2 , respectively.

\section{Acknowledgment}

We are grateful to the Mathematical Sciences Research Institute in Berkeley for support and hospitality.

\section{References}

[Cirre 2000] F. J. Cirre, "Automorphism groups of real algebraic curves which are double covers of the real projective plane", Manuscripta Math. 101:4 (2000), 495-512. MR 2001f:14055 Zbl 0984.14014

[Gross and Harris 1981] B. H. Gross and J. Harris, "Real algebraic curves", Ann. Sci. École Norm. Sup. (4) 14:2 (1981), 157-182. MR 83a:14028 Zbl 0533.14011

[Hartshorne 1977] R. Hartshorne, Algebraic geometry, Grad. Texts in Math. 52, Springer, New York, 1977. MR 57 \#3116 Zbl 0367.14001

[Huisman 1999] J. Huisman, "Real quotient singularities and nonsingular real algebraic curves in the boundary of the moduli space", Compositio Math. 118:1 (1999), 43-60. MR 2000i:14033 Zbl 0949.14017 
[Huisman 2000] J. Huisman, "Real Teichmüller spaces and moduli of real algebraic curves", pp. 145-177 in Real algebraic geometry and ordered structures (Baton Rouge, LA, 1996), edited by C. N. Delzell and J. J. Madden, Contemp. Math. 253, Amer. Math. Soc., Providence, RI, 2000. MR 2001d:32017 Zbl 0986.14037

[Huisman 2005] J. Huisman, "The space of unordered real line arrangements", Discrete Comput. Geom. 33:1 (2005), 157-163. MR 2006b:52021 Zbl 1078.52015

[Huisman and Lattarulo 2005] J. Huisman and M. Lattarulo, "Imaginary automorphisms on real hyperelliptic curves”, J. Pure Appl. Algebra 200 (2005), 318-331. MR 2006h:14039 Zbl 1077.14038

[Lattarulo 2003] M. Lattarulo, “The Schottky problem for hyperelliptic real curves”, Comm. Algebra 31:4 (2003), 1679-1703. MR 2004d:14034 Zbl 1059.14070

Received May 27, 2005. Revised January 21, 2006.

JOHANNES HUISMAN

DÉPARTEMENT DE MATHÉMATIQUES

LABORATOIRE CNRS UMR 6205

UFR SCIENCES ET TECHNIQUES

UNIVERSITÉ DE BRETAGNE OCCIDENTALE

6, AVENUE ViCTOR LE GORGEU

CS 93837

\title{
29238 BREST CEDEX 3
}

FRANCE

johannes.huisman@univ-brest.fr

http://stockage.univ-brest.fr/ huisman/

\author{
Michele Lattarulo \\ DIPARTIMENTO DI MATEMATICA \\ UNIVERSITÀ DI GENOVA \\ Via DODECANESO 35 \\ 16146 GENOVA \\ ITALIA
}

lattarul@dima.unige.it

http://www.dima.unige.it/ lattarul/ 